NISTIR 8338

\title{
Bridging Analog Land Mobile Radio to LTE Mission Critical Push-to-Talk Communications
}

Christopher Walton

Chic O’Dell 
NISTIR 8338

\title{
Bridging Analog Land Mobile Radio to LTE Mission Critical Push-to-Talk Communications
}

\author{
Christopher Walton \\ Chic O'Dell \\ Communications Technology Laboratory
}

This publication is available free of charge from:

https://doi.org/10.6028/NIST.IR.8338

December 2020

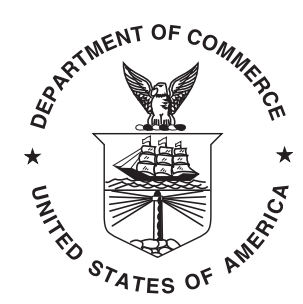

U.S. Department of Commerce Wilbur L. Ross, Jr., Secretary

National Institute of Standards and Technology Walter G. Copan, NIST Director and Under Secretary of Commerce for Standards and Technology 
Certain commercial entities, equipment, or materials may be identified in this document in order to describe an experimental procedure or concept adequately. Such identification is not intended to imply recommendation or endorsement by the National Institute of Standards and Technology, nor is it intended to imply that the entities, materials, or equipment are necessarily the best available for the purpose.

National Institute of Standards and Technology Interagency or Internal Report 8338

Natl. Inst. Stand. Technol. Interag. Intern. Rep. 8338, 33 pages (December 2020)

This publication is available free of charge from: https://doi.org/10.6028/NIST.IR.8338 


\title{
Acknowledgments
}

The authors would like to acknowledge technologists from both Nemergent Solutions SL and the University of the Basque Country for indispensable research guidance on Mission Critical Push-to-Talk (MCPTT) system design and integration.

\begin{abstract}
This paper explores a low-cost method to bridge analog public safety Land Mobile Radio (LMR) systems into a standards-based Long Term Evolution (LTE) Mission Critical Pushto-Talk (MCPTT) system. Design goals included robustness, cost, and the creation of a system that closely conforms to released and future standards. A proof of concept prototype that successfully bridges an analog LMR system with a standards-compliant LTE-based MCPTT system was developed and tested. The results and design details will be covered in this paper.
\end{abstract}

\section{Key words}

Digital Fixed Station Interface (DFSI); GNU Radio (GR); InterWorking Function (IWF); Inter-RF Subsystem Interface (ISSI); Land Mobile Radio (LMR); Long Term Evolution (LTE); Mission Critical Push-to-Talk (MCPTT); Mission Critical Voice (MCV); Open source VoIP software stack (PJSIP); Over the Air (OTA); Push-to-talk (PTT); Radio over Internet Protocol (RoIP); Software Defined Radio (SDR); Subscriber Unit (SU); User Equipment (UE). 


\section{Table of Contents}

Acronyms $\quad$ iv

1 Introduction 1

2 Background 1

2.1 LMR 2

2.2 3GPP MC Services 3

2.3 Interoperability Solutions 4

2.3.1 FSI 4

2.3.2 RoIP Gateway 4

2.3.3 ISSI/ CSSI

2.3.4 3GPP IWF 6

2.3.5 Over the Air Interfaces

3 Voice and Signaling Passthrough Client $\quad 8$

3.1 Software Defined Radio 9

$\begin{array}{ll}3.2 \text { GNU Radio } & 10\end{array}$

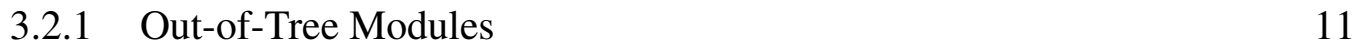

3.2.2 GNU Radio Audio and Signaling $\quad 12$

$\begin{array}{lll}3.3 \text { PJSIP } & 15\end{array}$

4 Test Bed $\quad 17$

$\begin{array}{lll}4.1 & \text { LMR Hardware } & 17\end{array}$

$\begin{array}{lll}4.2 & \text { MCPTT } & 18\end{array}$

$\begin{array}{llr}\text { 4.2.1 Enabler } & 19\end{array}$

4.3 LTE System Architecture Evolution 19

5 Results $\quad 20$

$\begin{array}{lll}5.1 & \text { Future Work } & 20\end{array}$

5.1.1 Accessibility and Reliability 21

5.1.2 Audio Handling 21

5.1.3 In-Band Signaling with Analog FM 21

\begin{tabular}{ll}
5.1 .4 & IWF Integration \\
\hline
\end{tabular}

$\begin{array}{ll}\text { References } & 23\end{array}$ 


\section{List of Figures}

Fig. 1 ISSI services architecture $\quad 5$

Fig. 2 Target IWF connection architecture 7

Fig. 3 Passthrough client system diagram 8

Fig. 4 FCC FM Narrow banding initiative 9

Fig. 5 Functional GNU Radio Architecture 10

Fig. 6 GNU Radio example gr-dsd flowgraph 11

Fig. 7 GNU Radio P25 channel breakout $\quad 12$

Fig. 8 GNU Radio downlink flowgraph section $\quad 13$

Fig. 9 GNU Radio uplink flowgraph section 13

Fig. 10 GNU Radio tagged data stream $\quad 14$

Fig. 11 PJSIP command line bare-bones MCPTT client 16

Fig. 12 Successful SIP registration flow sequence 16

Fig. 13 Client-based proof of concept test bed 17

Fig. 14 Nemergent UE MCPTT applications $\quad 18$

Fig. 15 Polaris EPC core architecture 19 


\section{Acronyms}

3GPP Third Generation Partnership Project. 3-8, 15, 18-22

5G Fifth Generation. 3

AFSI Analog Fixed Station Interface. 4

ALSA Advanced Linux Sound Architecture. 14, 20, 21

AMR-WB Adaptive Multi-Rate Wideband. 15, 16

ATIS Alliance for Telecommunication Industry Solutions. 6

CGRAN Comprehensive GNU Radio Archive Network. 11

CSSI Console Subsystem Interface. 2, 4, 5

D-STAR Digital Smart Technologies for Amateur Radio. 11

DFSI Digital Fixed Station Interface. i, 2, 4

DHS Department of Homeland Security. 22

DMR Digital Mobile Radio. 11

DSD Digital Speech Decoder. 11, 13

DSP digital signal processing. 8

E-UTRAN Evolved UTRAN. 19

eMBMS enhanced Multimedia Broadcast Multicast Services. 3

EPC Evolved Packet Core. 19

ETSI European Telecommunications Standards Institute. 5, 6

FCC Federal Communications Commission. 2, 9

FM Frequency Modulation. 1, 2, 8, 9, 13, 14, 17, 19-21

FSI Fixed Station Interface. 4

GR GNU Radio. i, 8, 10-15, 17, 21, 22

GRC GNU Radio-Companion. 10, 11

GUI Graphical User Interface. 16 
IP Internet Protocol. 4, 21

ISI Inter-System Interface. 5

ISSI Inter-RF Subsystem Interface. i, 1, 2, 4-6, 22

IWF InterWorking Function. i, 3-9, 17, 19-22

IWG InterWorking Group. 22

JLMRLTE Joint LMR and LTE. 6

KMS Key Management Server. 16

LMR Land Mobile Radio. i, 1-22

LTE Long Term Evolution. i, 1-3, 6-8, 10, 12, 14, 16, 17, 19, 20, 22

MC Mission Critical. 1, 3, 4, 6-9, 15, 16, 18-20, 22

MCData Mission Critical Data. 1, 3, 19

MCPTT Mission Critical Push-to-Talk. i, 1-4, 6, 8, 12, 14-22

MCV Mission Critical Voice. i

MCVideo Mission Critical Video. 1, 3

MCx Mission Critical Services. 3, 8, 22

NBFM Narrow Band FM. 9-11

NPSTC National Public Safety Telecommunications Council. 2, 21

NR New Radio. 3

OOT out-of-tree modules. 11, 12, 14

OTA Over the Air. i, 4, 8-10, 12, 15, 20

P25 Project 25. 2, 4-6, 11

PCM pulse code modulation. 11

PSCR Public Safety Communications Research. 1, 8, 12, 17-19, 22

PSTA Public Safety Technology Alliance. 4

PTT push-to-talk. i, 1, 4, 8, 9, 12, 14-16, 20, 22 
RFSS RF Sub-Systems. 5, 22

RoIP Radio over Internet Protocol. i, 4, 7

RTCP Real Time Control Protocol. 4, 22

RTP Real Time Protocol. 4, 5, 17, 19, 22

SDR Software Defined Radio. i, 4, 7-11, 13-15, 17, 20, 22

SIP Session Initiation Protocol. 5, 16, 17, 19, 22

SU Subscriber Unit. i, 5, 6, 14, 17-22

SWIG Simplified Wrapper and Interface Generator. 10

TCCA The TETRA and Critical Communications Association's. 2

TCCE TETRA and Critical Communications Evolution. 6

TETRA Terrestrial Trunked Radio. 2, 5, 6

TIA Telecommunications Industry Association. 2, 4-6, 22

UE User Equipment. i, 18, 19

UHD Universal Software Radio Peripheral (USRP) hardware driver. 11, 13, 15

UHF Ultra High Frequency. 9, 17

URI Universal Resource Identifier. 16

VHF Very High Frequency. 9, 17

VoIP Voice over IP. 15, 22

WG6 Working Group 6. 3

WTSC Wireless Technologies and Systems Committee. 6 


\section{Introduction}

Land Mobile Radio (LMR) is the leading communications technology used by public safety for push-to-talk (PTT) communications. With the widespread deployment and desirable features of nationwide broadband networks, there are significant benefits to be gained by augmenting or, in the far future, replacing current public safety communications methods with modern Long Term Evolution (LTE) communications technologies. Emerging Mission Critical (MC) standards will provide public safety with Mission Critical Push-toTalk (MCPTT), Mission Critical Data (MCData), and Mission Critical Video (MCVideo) capabilities above and beyond what current LMR is able to provide. The possibility of including MCData and MCVideo into a nationwide public safety broadband communications network stands to introduce profound changes in the tools available to the public safety community.

Given that LMR continues to be the primary communications solution for public safety, the ability to interface with both broadband and other LMR systems is vital to maintaining a robust and interoperable public safety communications ecosystem. Additionally, highly interoperable solutions will help ease the transition to broadband technologies. Both LMR and LTE systems can be run in parallel, and in many cases they can complement one another. Interworking technologies for digital LMR systems exist in the commercial world and are being actively developed. In the case of antiquated but still widely deployed conventional analog LMR, MCPTT interworking technologies are not being developed at the same rate as their digital system counterparts. Therefore, analog LMR has the potential to be excluded from interacting with both Inter-RF Subsystem Interface (ISSI)-based LMR and broadband MC systems.

Responding to these challenges and opportunities, Public Safety Communications Research (PSCR) staff initiated the Bridging Analog LMR to LTE Communications project. This project has the goal of investigating and developing technologies that will bridge conventional analog Frequency Modulation (FM) LMR with MCPTT-enabled broadband LTE. Research was conducted with three governing metrics in mind: robustness, standards compliance, and cost. Any interoperability solution should meet or exceed uptime performance for similar public safety sector equipment. Standards compliance is vital to a healthy multi-vendor communications ecosystem. The expense of available interoperability solutions is cost prohibitive to many public safety groups. This paper serves to illustrate new tools and methodologies for LMR to LTE interoperability research, based on the metrics outlined above.

\section{Background}

Public safety is a primary user in a community of MCPTT users that are being impacted with the rapid change and creation of new technologies. Historically, public safety has re- 
lied on LMR but, with the advent of widely available and dedicated access to broadband networks, an undeniable technical migration is taking place. This migration will not take place as a discrete jump from legacy to new technologies, but as a gradual transition. To accomplish either an aggressive or conservative migration strategy, interoperability between the new and old technologies is necessary.

Radio interoperabilty solutions existed before the need to bridge LMR and LTE. These technologies can be used as starting points to develop MCPTT bridging solutions. Current bridging solutions have varying degrees of standards compatibility amongst several standards bodies. Standards-based solutions are in development, but an understanding of currently available solutions is needed to make any future standards as robust and widely acceptable as possible.

\subsection{LMR}

Based on available data, the number of FM LMR systems in use is unclear. Many rural and urban cites still rely on analog FM-based LMR public safety communications. Therefore, we are operating under the assumption that analog LMR sites will continue to constitute an appreciable share of public safety communications. Interoperability solutions naturally target more modern digital LMR systems. These digital interoperability methods are often completely incompatible with analog LMR. Digital LMR interoperability solutions take advantage of ISSI, Console Subsystem Interface (CSSI), or Digital Fixed Station Interface (DFSI). These solutions do not support analog LMR systems. Analog LMR is an older technology and therefore not at the forefront of interworking development. This has resulted in a market gap for analog interoperability solutions. This paper will explore possible technologies and solutions to fill the gap.

The first obstacle when developing an interworking function is the lack of standards describing the specific operation of conventional analog FM LMR systems. For example, pure analog systems cannot support data services without augmentation like in-band signaling. There are many in-band signaling schemes used in analog LMR. Each signaling schema varies in its implementation and supported features, as shown in Telecommunications Industry Association (TIA) Standard 102.E [1].

Digital LMR standards can be used to expand communication architecture and support public safety analog use cases. TIA's digital LMR standard provides guidance about interoperability, implementation, and feature sets for Project 25 (P25) LMR systems. The TETRA and Critical Communications Association's (TCCA) Terrestrial Trunked Radio (TETRA) digital LMR standard provides yet another framework that can be referenced to understand LMR system operation.

Working groups and federations provide additional specific expertise for public safety LMR communications. One such entity is National Public Safety Telecommunications Council (NPSTC), who has published a comprehensive report [2] providing an exhaustive overview of public safety communications use cases, features lists, and technical requirements. Further characterization is available from the Federal Communications Com- 
mission (FCC) frequency allocation rules discussed in Sec. 3.1. Outside of digital LMR standards, Third Generation Partnership Project (3GPP) MCPTT standards specifically address LTE MCPTT interworking to LMR, which will be discussed at length in Sec. 2.3.4.

Taking advantage of the above resources provides information concerning use cases, feature sets, and interoperability methods for LMR systems. Although some of these resources address interoperability to other LMR systems, none are able to give a complete end to end description for interworking between broadband LTE MCPTT systems. Analog LMR will never be able to support the full feature set published by $3 \mathrm{GPP}$ as defined in the MCPTT standard. Even with analog's inherent limitations, a simple reliable voice patch is valuable. Possible augmentations to analog systems that allow additional functionality will be discussed in Sec. 5.1. As presented above, there is a wealth of documentation concerning system operation and interworking methods between LMR and LTE.

\subsection{GPP MC Services}

The augmentation of LMR communications with Mission Critical Services (MCx) has proven problematic despite strong $\mathrm{MC}$ standards and a clear need in the public safety community for updated technology. There are many factors that inhibit adoption of new technologies like network reliability, hardware availability, coverage concerns, human elements, etc. Interworking/interoperability has been identified as a mechanism to facilitate a more gradual adoption of 3GPP-enabled technologies in the public safety arena. The ability to communicate between these systems will enable public safety users with new tools to save lives and provide system redundancy.

In 2016, MCPTT was added by 3GPP's SA Working Group 6 (WG6) Release 13 [3]. This release enabled MC communications over LTE with priority and preemption. Release 14 [4] continued to add features to the MCPTT standard such as enhanced Multimedia Broadcast Multicast Services (eMBMS), MCVideo, and MCData. Per 3GPP Release 15 [5], frozen Q3 2019, MC-services have been included in the Fifth Generation (5G) standard, and the InterWorking Function (IWF) was defined. Looking forward to 3GPP Release 17, scheduled through 2021, WG6 meeting notes show that MC services are still actively being discussed and added into 5G New Radio (NR) architecture. It is clear that the 3GPP MC service standards will be adopted not only by public safety but also by other emerging $5 \mathrm{G}$ commercial sectors.

A major push to implement MC services in the U.S. has been through the First Responder Network Authority (FirstNet). FirstNet was created through government legislation with a charter to establish, operate, and maintain an interoperable public safety broadband network [6]. FirstNet has adopted the 3GPP MC standard and has committed to hosting multiple MCPTT vendors on its network [7, 8]. Looking towards the future, FirstNet has committed to providing interoperability between LMR and MCPTT [9]. 


\subsection{Interoperability Solutions}

Currently, Fixed Station Interface (FSI), Radio over Internet Protocol (RoIP), and ISSI/ CSSI are existing solutions commonly used to implement interoperability from one LMR system to another. One of the largest proponents of LMR technology development has been the defense sector, where there has been a huge push for communications systems research and development. This effort has greatly advanced the state of Software Defined Radio (SDR) and has shown that Over the Air (OTA) interfaces are viable LMR connection points that are often overlooked in commercial passthrough solutions [10]. 3GPP Releases 15 and 16 have complete architecture and protocol specifications for MCPTT interworking with LMR systems via 3GPP IWF. This new interoperability tool will provide a passthrough to the host $\mathrm{MC}$ application server, allowing access to the $\mathrm{MC}$ services ecosystem. These technologies will be further discussed in the following subsections.

\subsubsection{FSI}

TIA's P25 standard defines the FSI for digital LMR systems [11]. The Analog Fixed Station Interface (AFSI) provides audio and control signaling between two connected systems by passing an analog voice signal. Control signaling is accomplished by adding in-band signaling to the transmitted AFSI analog channel. TIA has defined tone remote control as a general standard to accomplish in-band signaling. Unlike AFSI, DFSI operates with an Internet Protocol (IP)-based methodology, using Real Time Protocol (RTP) and Real Time Control Protocol (RTCP) for voice and data transmission. DFSI has been earmarked by the Public Safety Technology Alliance (PSTA) interoperability subcommittee as an option for future integration of analog LMR to IWF [12].

\subsubsection{RoIP Gateway}

A much lower cost interoperability solution that is commercially available and compatible with all types of common LMR systems is a RoIP gateway. This technology uses a donor PTT radio registered and affiliated to the host LMR system. The donor radio handles all LMR system specifics and provides the RoIP solution with raw audio. By using the donor radio, the RoIP gateway does not need to know anything specific about either LMR endpoint. This solution largely bypasses vocoding, encryption, and group management issues. A highlight of RoIP solutions is their ability to be deployed quickly from a console or in the field. As with other LMR interoperability technologies or 3GPP's IWF, RoIP technology uses IP protocol to connect target endpoints.

There are some serious drawbacks to RoIP interoperability solutions. RoIP is not governed by an open standard and this point should not be overlooked. Using nonstandard solutions inhibits compatibility, limits market share, and makes quality control difficult. In the absence of a RoIP standard, gateway features vary between vendors. Most solutions do not support location services or unit identifier data passed between systems. Although using RoIP is much cheaper than ISSI, the donor radios necessary for RoIP operation 
are an expensive and finite public safety resource. Furthermore, each gateway supports one communications channel. In a worst-case scenario between two conventional LMR systems, in order to support $n$ communications channels, $n$ times two gateways and donor radios are needed. The increase in necessary donor radios does not lend itself to costeffective scalability.

\subsubsection{ISSI/ CSSI}

ISSI and CSSI are part of the TIA P25 standard and can connect multiple RF SubSystems (RFSS), allowing communication across otherwise disparate trunked P25 LMR systems. TETRA, the European digital radio standard, uses the Inter-System Interface (ISI) standardized by European Telecommunications Standards Institute (ETSI). A digital LMR system containing one of these interoperability solutions (ISSI/ CSSI or ISI) is currently without a completed interworking standard to facilitate standards-compliant connections to 3GPP's IWF. The development of ISSI-to-IWF signaling will be further discussed in Sec. 2.3.4.

A TIA standard ISSI uses Session Initiation Protocol (SIP) and RTP for call control and voice transport. Using these protocols, the ISSI is able to support group calls, Subscriber Unit (SU) to SU calls, and P25 data across connected systems. When making calls through an ISSI, special call parameters can be requested. These ISSI features include confirmed/unconfirmed transmission mode, transmission trunked, or message calls and support of multi-site LMR public safety use cases. Confirmed transmission mode through the ISSI allows a call to be placed only when all users are available to the system. Unconfirmed call mode will place the call in a best effort capacity regardless of $100 \%$ user availability. The ISSI itself is made up of 4 functional entities as seen in Fig. 1. Because ISSI solutions have deep hooks into their host LMR system services architecture, they are able to offer interoperability features other solutions have no way to implement.

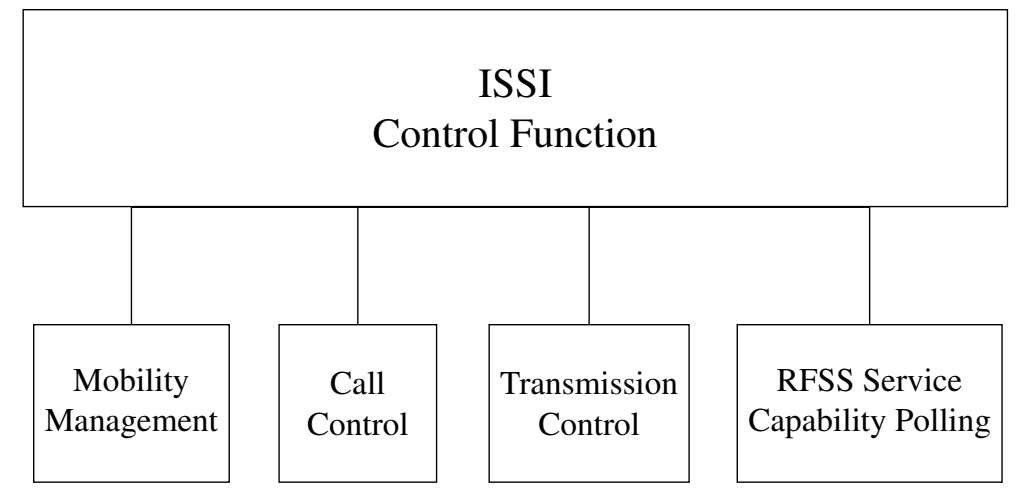

Fig. 1. ISSI services architecture 
It is worth noting that implementation of an ISSI takes significant time and setup effort at both system endpoints. This situation can be exacerbated if the system endpoints are provided by different vendors. Although ISSI is standard-compliant and the most capable commercial bridging technology available, it is cost prohibitive for many agencies. Complexity of the solution requires that deployment and configuration work be performed by the commercial entity producing the ISSI. Regardless of the completeness of the LMR standard or the budget of the public safety organization, ISSI is incompatible with analog LMR.

\subsubsection{GPP IWF}

3GPP Releases 15 and 16 have defined architecture and protocol specifications for interworking with LMR systems via the IWF. The IWF provides interfaces that support encryption, group management, and transcoding of interconnected MC and LMR systems. MCPTT application server functionality is implemented within the IWF, which exists as a participating server associated with the host MCPTT-enabled LTE system. The LMR connection to the IWF has been deemed out of scope by $3 \mathrm{GPP}$ and therefore has been left to respective LMR standards bodies. These LMR standards are currently under development. When completed, the IWF will provide a passthrough for LMR SUs to LTE MC clients.

LMR standards for IWF connections are being developed in a joint effort between TIA and Alliance for Telecommunication Industry Solutions (ATIS). The Joint LMR and LTE (JLMRLTE) working group is comprised of members of ATIS's Wireless Technologies and Systems Committee (WTSC) and TIA. TETRA LMR systems are governed by ETSI and TETRA and Critical Communications Evolution (TCCE), who are working to define these interfaces for European public safety. After these efforts have been completed, there will be a standards-compliant implementation of the interfaces necessary to connect LMR and the IWF. Conventional P25, trunked P25, and analog interfaces have been preliminarily defined as L102-C, L102-T, and L603, as shown in Fig. 2. 


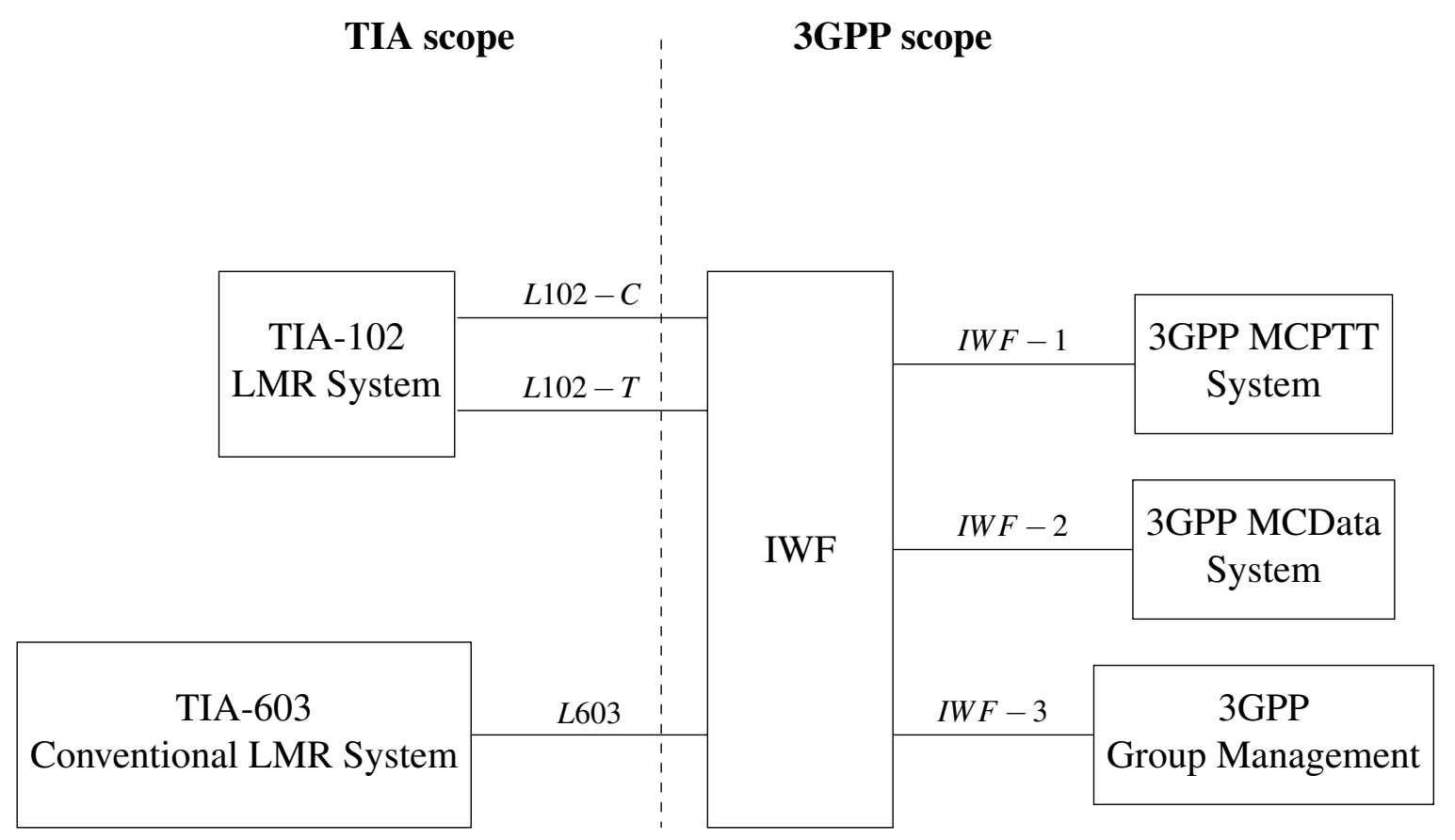

Fig. 2. Target IWF connection architecture

3GPP IWF documents $[13,14]$ provide a description of suggested IWF architectures. 3GPP clearly states, "The IWF, along with its LMR system, will appear as a peer interconnected MC system." We understand from this declaration that the IWF will provide any necessary translations between the LMR and MC systems. Until standards-compliant IWFs become commercially available, it is necessary to find workarounds to facilitate immediate testing and development.

\subsubsection{Over the Air Interfaces}

SDR technology has not been widely adopted in LMR to LTE interoperability solutions. This is positioned to change with the continued advancement in SDR technologies and computing hardware, making SDR a prime research target for communications bridging technologies. When paired with software able to interface and control the SDR, new and exciting communications solutions are possible. For example, the replacement of multiple RoIP donor radios with a single SDR solution provides the possibility of significant improvements upon existing products. SDR has the ability to provide coverage over multiple channels of an LMR system, compared with the single channel supported per donor radio. SDR excels in two main areas: real-time RF transceiver flexibility and software-controlled modulation. Both of these are key abilities when bridging a wide range of disparate communications systems. Additionally, the physical attributes of SDR solutions are often considerably smaller, lighter, and more power efficient than standard LMR handhelds. Use cases in the space and defense sector show successful implementation of high reliability 
SDR communication applications. PSCR's implementation of SDR in our OTA analog LMR-to-broadband MCPTT interoperability solution will be discussed in Sec. 3.1.

\section{Voice and Signaling Passthrough Client}

The high-level system diagram shown in Fig. 3 identifies three fundamental technologies that make up the PSCR interoperability solution (herein referred to as the passthrough client): SDR, GNU Radio (GR), and the PTT client. Together, these technologies can provide basic interoperability between a conventional analog FM LMR system and an LTE-enabled MCPTT. The first of these steps, SDR, is used to monitor and interact with the OTA interfaces at the LMR system endpoint, feeding that data into GR. Second, GR commands all RF interfaces, handles real-time digital signal processing (DSP), and generates LMR floor control signaling. Finally, a PTT client is implemented as a non-MCx user at the MCPTT application server endpoint. Once implemented, the passthrough client allows either system endpoint to place and receive basic calls to, or from, the otherwise disconnected communications system.

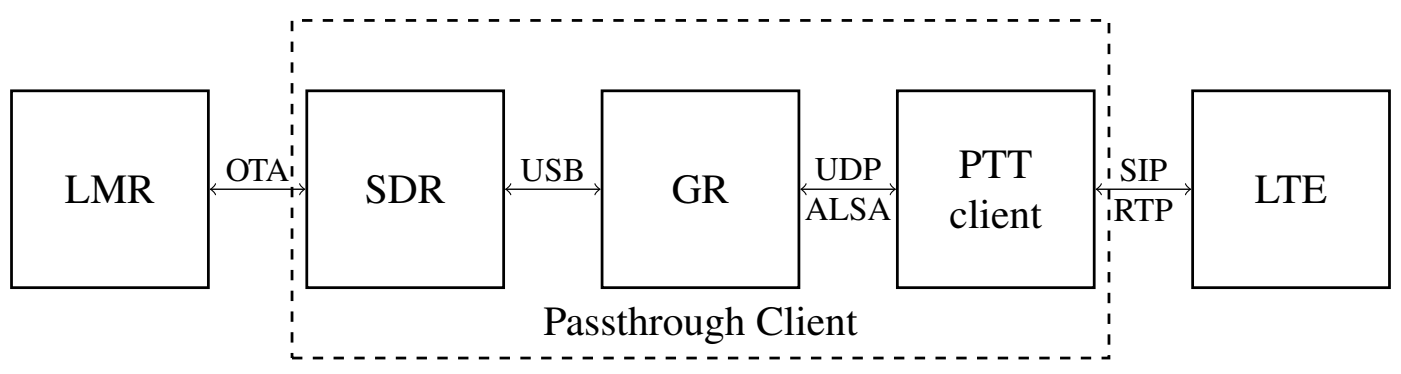

Fig. 3. Passthrough client system diagram

Implementation of the passthrough client solution requires one computing platform that runs all three bridging resources identified above. This computing platform must have an IP connection to the MCPTT-enabled LTE system endpoint. Additionally, the SDR must be within coverage of the LMR system endpoint. Sec. 4 will further discuss specifics of the PSCR test bed.

The first and biggest divergence made from the 3GPP standard is the connection of our passthrough client directly to the MCPTT application server. The standard method prescribed by $3 \mathrm{GPP}$ requires the client connect to an IWF, not directly to the MCPTT application server [15]. The LMR connection to the IWF, shown in Fig. 2, has not been fully defined, and there are no known commercial off-the-shelf IWF solutions available for purchase. An IWF is a nontrivial piece of software; its creation is beyond the scope of this project. In short, the decision to connect the passthrough client directly to the MCPTT application server was made out of necessity. Once a standards-compliant IWF becomes available, our passthrough client will connect to the IWF, not directly to the MCPTT application server. The passthrough client provides a functional, albeit nonstandard, proof of concept research platform to develop and become familiar with the MC standard call 
types, MCx features, and eventual IWF integration. Client usage with IWF is further discussed in Sec. 5.1.4.

\subsection{Software Defined Radio}

As discussed in Sec. 2.3.5, SDR allows for exciting new approaches to communications bridging technologies in the public safety arena. PTT radio interworking/interoperability solutions are often siloed by vendor and can be orders of magnitude more expensive than SDR-based solutions. With a frequency-compatible SDR and the correct software, we have found it is possible to establish OTA interfaces with many different LMR systems. Moreover, an OTA interface allows great freedom in the location of the passthrough client as the physical transceiver simply needs to be in the coverage area of the LMR system endpoint.

Per FCC regulation, it is most common to find analog FM LMR systems operating in the Very High Frequency (VHF)/ Ultra High Frequency (UHF) frequency range. As of 2013, the FCC has mandated that all public safety communications systems in the VHF/ UHF spectrum use Narrow Band FM (NBFM) [16]. NBFM is a channel mask cutting down the previous $25 \mathrm{kHz}, \mathrm{FM}$ bandwidth channels to $12.5 \mathrm{kHz}$ bandwidth. The FCC has stated that it will, in the future, mandate a channel band of $6.25 \mathrm{kHz}$.

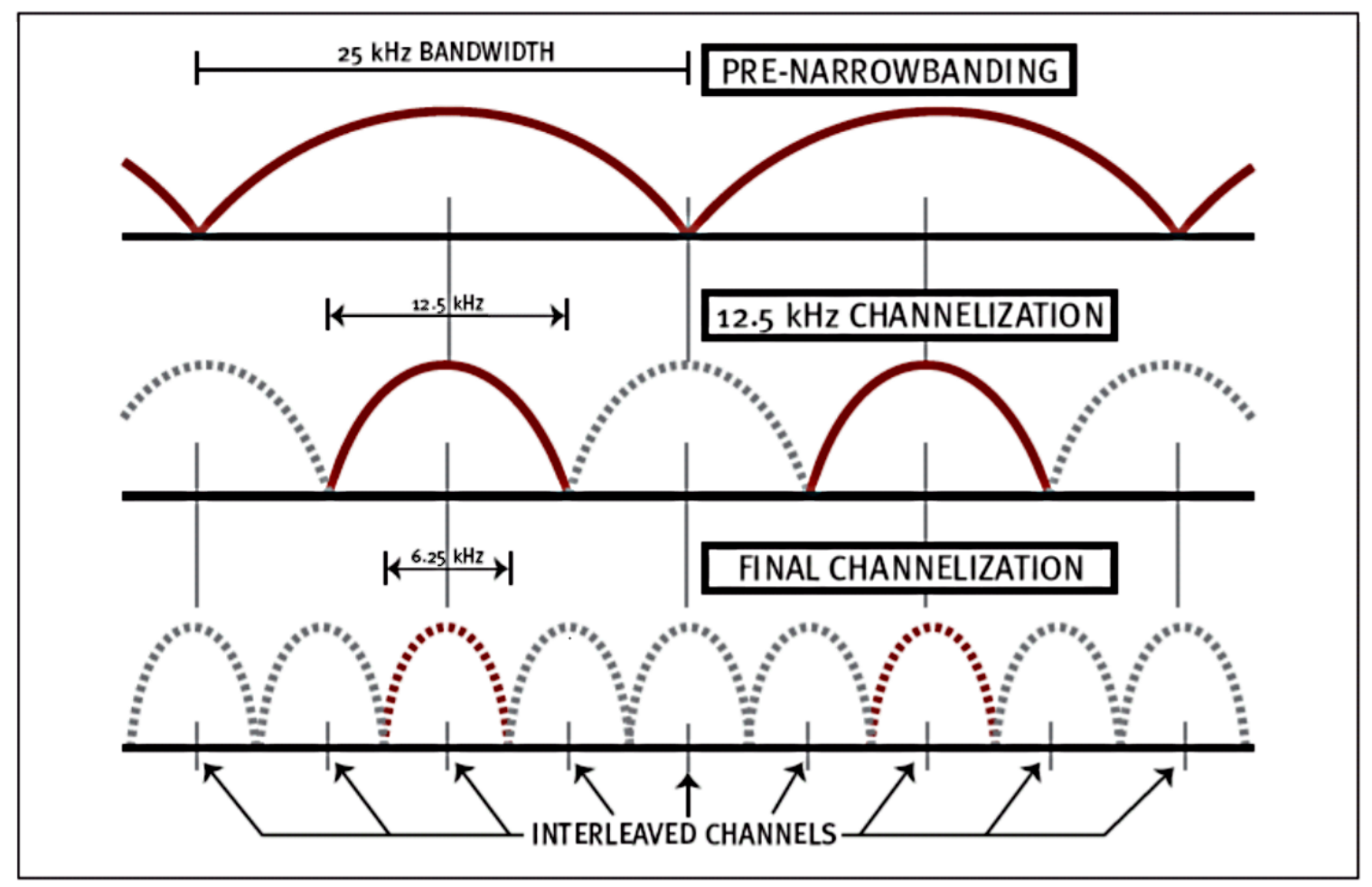

Fig. 4. FCC FM Narrow banding initiative

Given the bandwidth constraints imposed by the FCC [17], seen in Fig. 4, current 
generation SDR specifications are more than capable of supporting many simultaneous OTA LMR channels. Commonly available SDR products are able to receive and transmit in frequency ranges easily covering most FCC-mandated LMR band allocations from 100 $\mathrm{MHz}$ to $860 \mathrm{MHz}$ [18]. Given a common LMR base station is capable of supporting 100 channels using $150 \mathrm{kHz}$ channel spacing, simple calculations show that an off-theshelf SDR needs approximately $15 \mathrm{MHz}$ of instantaneous bandwidth to accommodate 100 continuous NBFM communications channels.

One commercially available SDR, the Ettus Research B210, has published benchmarks documenting $56 \mathrm{MHz}$ of instantaneous bandwidth with continuous frequency coverage from $70 \mathrm{MHz}$ to $6 \mathrm{GHz}$ [19]. Therefore, it can be expected that one B210 SDR is capable of handling over 100 simultaneous NBFM LMR channels through the use of broad spectrum capture. The Ettus Research B210 has ample bandwidth to support this application.

\subsection{GNU Radio}

GR is a long-standing open-source software project that has developed in tandem with SDR. GR allows SDR to be leveraged as a dynamically configurable RF back end while also providing real-time signal processing and a graphical user front end. GR itself is used for real-time data processing, and pairing it with SDR allows design of customized signal processing applications. Example implementations of GR and SDR technologies are widespread: LTE, LMR, radar systems, satellite ground stations and many more custom RF solutions [20]. GR enables its community with documentation, tools, and example implementations ensuring continued project development and support.

Development of GR communications solutions most commonly uses the GNU RadioCompanion (GRC) graphical interface. GRC is bundled into the GR package install, providing the user with tools and a space for flowgraph development. The flowgraph defines GR run-time operation, outlining connections of signal processing blocks through which data is streamed. Once a flowgraph has been created, GRC can generate the python flowgragh equivalent code and execute it. Functional block code is usually written in $\mathrm{C}++$ but can also be written in Python. For blocks written in $\mathrm{C}++$, Simplified Wrapper and Interface Generator (SWIG) provides a Python binding, enabling usage in exclusively Python-based flowgraphs. A reference architecture is shown in Fig. 5.

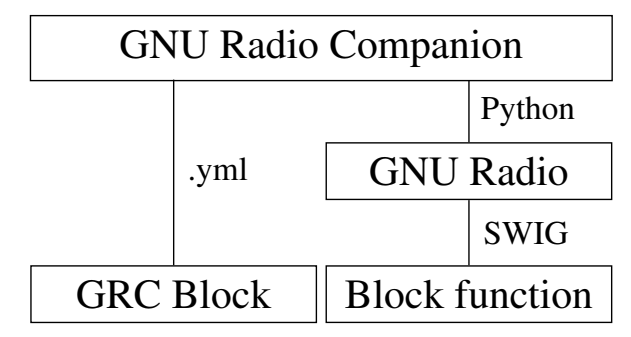

Fig. 5. Functional GNU Radio Architecture 


\subsubsection{Out-of-Tree Modules}

Custom modules serve to augment the base block offering of GR and are referred to as out-of-tree modules (OOT). The gr modtool utility [21], included in the install of GR, is used to build new OOTs. Gr modtool provides a bare-bones module and block generator, allowing the developers to manage their modules. Once installed alongside GR, the OOT provides the user access to custom blocks and flowgraph implementations contained in the module through GRC. Custom modules provide the GR community a way to collaborate and disseminate code based around specific projects. The Comprehensive GNU Radio Archive Network (CGRAN) [20] is a source code repository of popular community modules showing the wide range of possible GR applications.

A pertinent example of a useful GR OOT for the public safety community is Digital Speech Decoder (DSD), hosted on CGRAN as $g r-d s d$ [22]. This OOT provides audio output for a given unencrypted P25, Digital Mobile Radio (DMR), or Digital Smart Technologies for Amateur Radio (D-STAR) input stream. As discussed in Sec. 3.1, the instantaneous bandwidth of a common SDR is large when compared to NBFM LMR systems. Therefore, it is possible to receive many or all conventional channels of a given LMR site using one SDR capture. GR allows this broadband capture to be broken down into NBFM channels delivering raw audio to the user. Fig. 6 shows a single-channel decode GRC flowgraph using the DSD OOT.

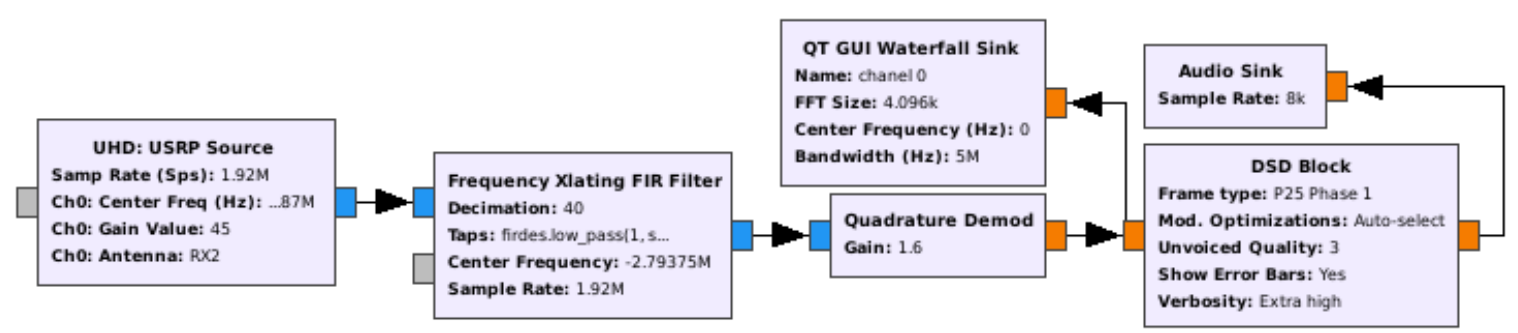

Fig. 6. GNU Radio example gr-dsd flowgraph

The flowgraph above begins with the UHD: USRP Source block. The Universal Software Radio Peripheral (USRP) hardware driver (UHD) is a common SDR driver that supports off-the-shelf Ettus Research SDR products. In GRC, the UHD provides both source and sink blocks, which facilitates SDR receive and transmit, as well as run-time and RF setting control. These settings include SDR sample rate, bandwidth, center frequency, etc. The UHD source block injects the SDR capture into the Frequency Xlating FIR Filter, thereby isolating the NBFM channel out of the broadband SDR capture. The now isolated LMR channel is fed into a demodulation block and then passed into DSD. The DSD Block converts the demodulated data stream into usable pulse code modulation (PCM) audio. This channelization, demodulation, and decode can be executed in parallel for all LMR channels in the broadband SDR capture. Fig. 7 shows this process used on three voice NBFM P25 LMR channels. It should be noted that the DSD project does not support 
trunked communication, but it will still output audio as decoded from the channel.
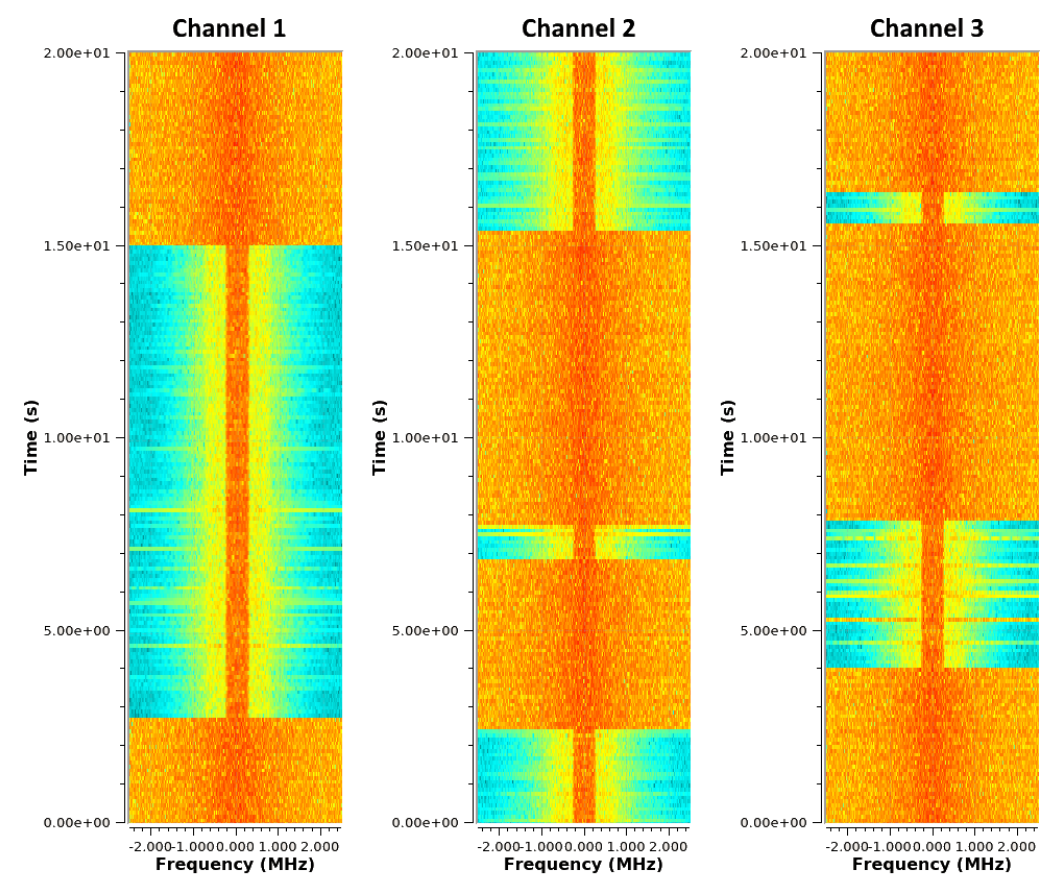

Fig. 7. GNU Radio P25 channel breakout

\subsubsection{GNU Radio Audio and Signaling}

For use in PSCR's analog LMR to LTE interoperability solution, a custom GR OOT module $g r$-LMR2LTE has been created. The gr-LMR2LTE module contains only one block, jsock, providing the necessary floor control signaling to the PTT client, as discussed in Sec. 3.3. The flowgraph used in our test bed is broken into two sections shown as downlink (Fig. 8) and uplink (Fig. 9). Both downlink and uplink sections are run in the same GR flowgraph, but they are separate and do not interact. The downlink section handles OTA communications from the LMR system endpoint toward the MCPTT LTE system. The uplink section interfaces with the passthrough client exchanging floor control and audio from the MCPTT LTE system and transmits MCPTT call audio onto the directed LMR channel. As stated above, the OOT-provided jsock block is used to create a network interface with the PTT client, discussed further in the following sections. 


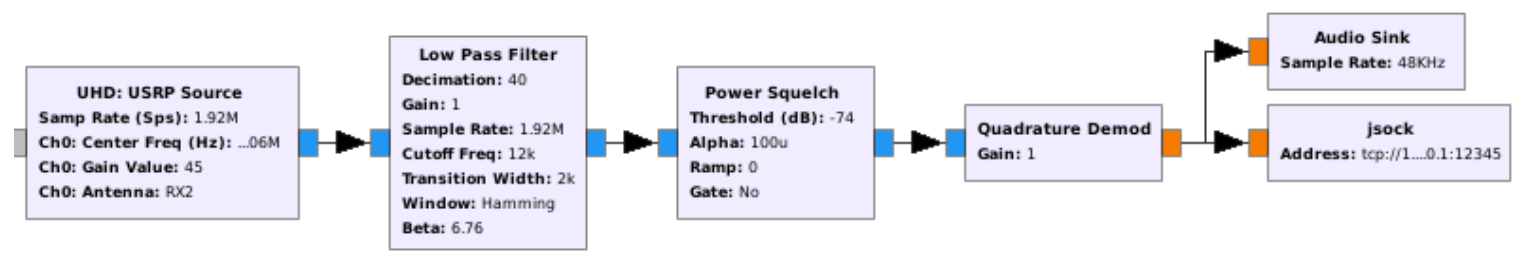

Fig. 8. GNU Radio downlink flowgraph section

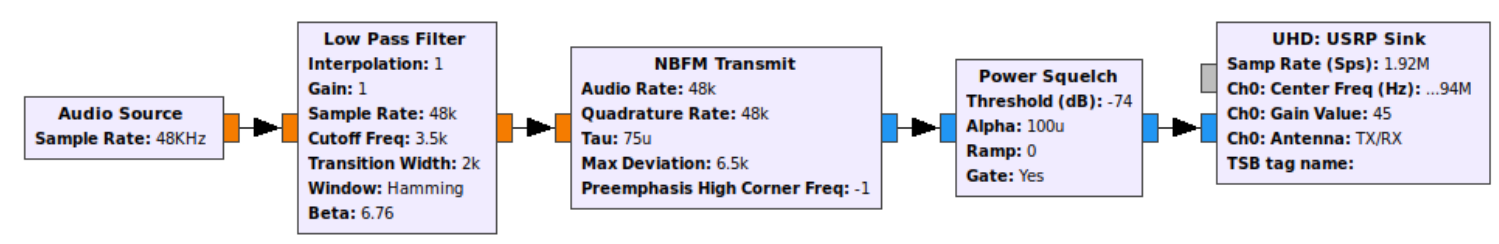

Fig. 9. GNU Radio uplink flowgraph section

Walking through the downlink flowgraph section, similar to the DSD implementation, the UHD block handles all communication between the SDR and GR. Our test bed only provides one conventional analog FM LMR channel and thus presents no need to channelize the SDR capture. The UHD block passes the captured channel data stream through a low pass filter and into a squelch. The power squelch block is important, as it is used to tag the data stream, marking the squelch triggers and releases. This tagging mechanism provides real-time LMR floor state information during passthrough client operation. An example of a tagged data stream can be seen in Fig. 10, where the starting tag is labeled as squelch_sob and the ending tag as squelch_eob. Squelch block settings allow for control of start, end, or burst tagging. The tagged data stream is demodulated and passed through another low pass filter for added noise suppression. 


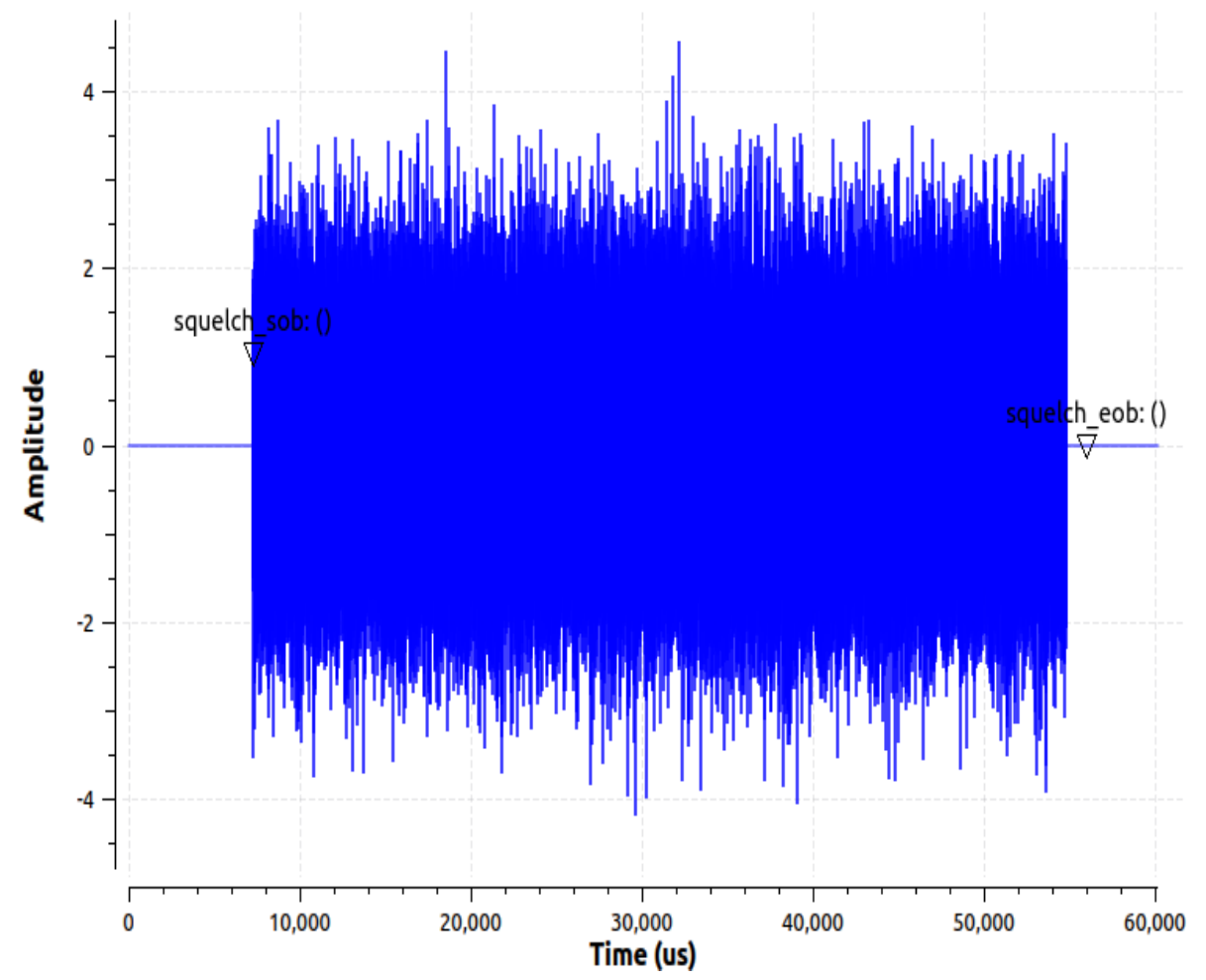

Fig. 10. GNU Radio tagged data stream

The data stream is terminated at Audio and jsock sink blocks, ending the downlink section of the passthrough client flowgraph. There are many different options when handling audio at this stage, and they will be discussed more fully in Sec. 5.1.2. For this proof of concept we have decided to use the audio architecture present on our GR computing platform, Advanced Linux Sound Architecture (ALSA). The GR Audio sink block passes its data stream into the audio infrastructure of the host sound architecture. This design allows any LMR communication detected by the SDR to be heard at the output of the GR flowgraph, and provides the PTT client access to the audio stream used in LMR to LTE calls.

The jsock sink block is a gr_modtool-generated OOT that passes floor control signaling from the GR flowgraph to the PTT client. On flowgraph start, the jsock block uses the open-source ZMQ library to build a publish-socket for subscription by the PTT client. Jsock looks for tags placed in the GR stream by the squelch block. Upon detection of a squelch tag, the jsock block publishes updated LMR floor control information over the socket to the subscribed PTT client. Through this exchange, the PTT client is notified whenever an LMR SU is keyed or de-keyed.

Fig. 9 shows the uplink portion of the GR flowgraph handling the LTE to LMR passthrough. In this portion of the flowgraph, no OOT blocks are used. Similar to the downlink section, ALSA is used to inject the LTE MCPTT client audio stream into GR via Audio source block. The audio stream is filtered, FM modulated, filtered again, and 
then passed into a squelch block. In the uplink instance, the squelch is not implemented to tag the data stream but to start or stop it completely. Notice the gate variable of this squelch block is set to true, opposite the setting of the squelch block used in the downlink flowgraph section. If true, the gate setting halts the data flow unless squelch conditions are met. A false gate pads the data stream with zeros instead of halting when the squelch is not triggered. Ending the uplink portion is a UHD sink that commands SDR transmission of the input data stream through SDR into the LMR endpoint. When the uplink squelch changes from active to squelched, the downstream UHD block will be provided with bursts of data. This periodic data stream is transmitted into the LMR endpoint by the UHD. If a burst transmission method were not implemented, the UHD sink would be fed a constant data stream, causing the SDR to continuously transmit. As part of the full passthrough client solution, both uplink and downlink portions of the presented flowgraph work together providing the necessary translation between the LMR OTA interface and the PTT client.

\subsection{PJSIP}

There are commercial MCPTT clients available for use today but, to interface the client with GR, we must be able to modify the source code. We were unable to find any opensource MCPTT clients suited for this use case; there is, however, a plethora of open-source Voice over IP (VoIP) client projects. These projects were used as a starting point providing client source code which was augmented and leveraged to support 3GPP-defined MCPTT client behavior.

PJSIP was the open-source project chosen to implement the necessary interfaces for the PTT client used in the passthrough client solution. PJSIP provides a free and opensource collection of standards-compliant communications libraries that are highly portable, feature rich, and well documented [23]. During PJSIP's build and install process, configuration options allowed for the addition of the Opencore-amr library, thereby providing PJSIP Adaptive Multi-Rate Wideband (AMR-WB) vocoding encode/decode capabilities. Out of the box, PJSIP provides all necessary components to run a VoIP client including sample source code. The VoIP client sample served as a solid starting point for further development toward a non-MCx client behavior for use in the passthrough solution, referred to herein as PTT client. The PTT client serves as a command line-based, bare-bones non-MCx entity, seen in Fig. 11. 


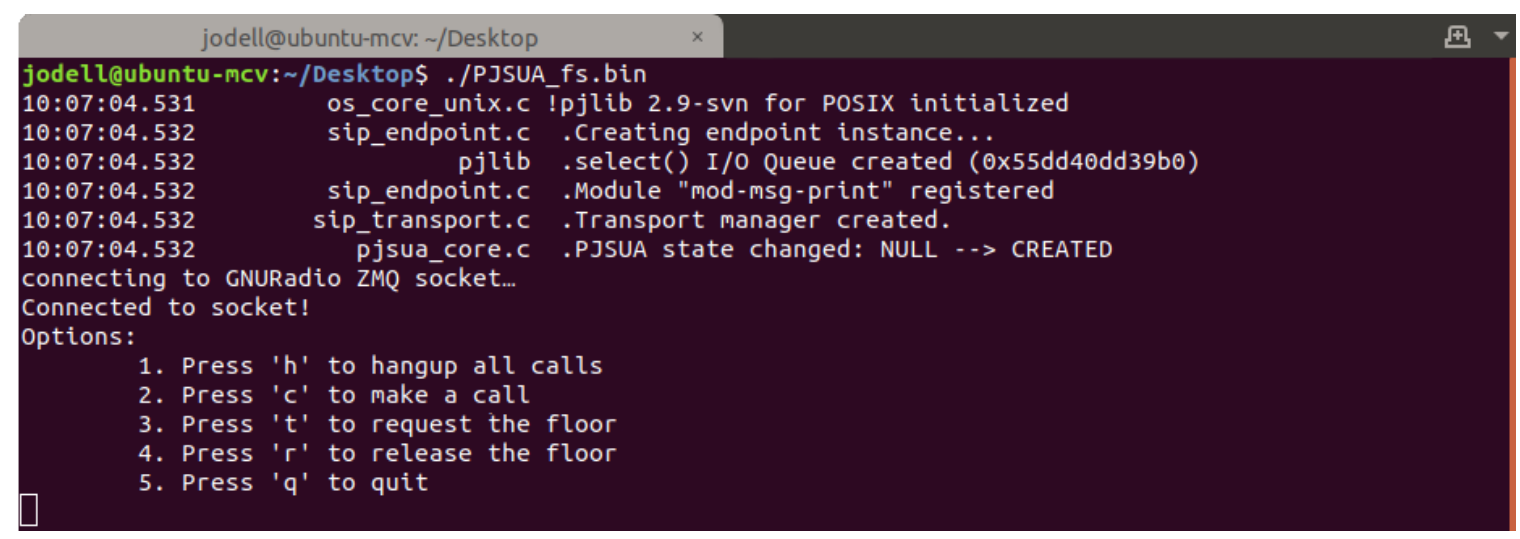

Fig. 11. PJSIP command line bare-bones MCPTT client

On startup, the MCPTT client uses its hardcoded SIP Universal Resource Identifier (URI) to perform SIP authentication to the MCPTT server endpoint, seen in Fig. 12. The PSCR LTE test bed does not include any Key Management Server (KMS), simplifying client initialization. There must be a dedicated non-MCx user provisioned on the MCPTT system endpoint for the PTT client to register successfully. This entry will define many attributes of the client on the MCPTT system: priority, vocoder selection, group membership, etc. Using the MCPTT operations and maintenance Graphical User Interface (GUI), we are able to directly control the passthrough client settings on the MCPTT system. Once authenticated on the MCPTT domain, the client behaves as any other non-MCx client. The passthrough client will wait to auto-answer any MCPTT call or place an LMR call.

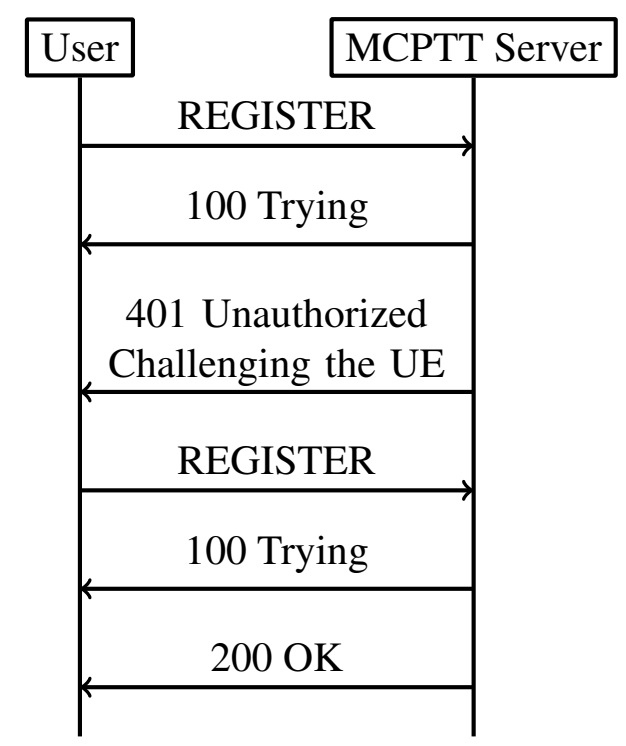

Fig. 12. Successful SIP registration flow sequence

During calls, the PTT client handles all AMR-WB vocoding used by the LTE system. Our passthrough client does not use encryption for call sessions, although PJSIP supports 
this capability. During MCPTT calls, floor control is achieved via SIP messaging directly to the MCPTT application server. The need for non-standard messaging will be eliminated with the addition of the IWF.

When an LMR portable is keyed, the MCPTT client is triggered via GR signaling as discussed in Sec. 3.2.2. Once triggered, the MCPTT client will attempt to place a private MCPTT call through the MCPTT application server using SIP and RTP protocols to set up sessions and transport audio. The MCPTT call type and destination are preconfigured but can be changed during MCPTT client operation. If the MCPTT server accepts the MCPTT client's request, the client will then place a private MCPTT call using the audio stream provided by the GR downlink flowgraph (Fig. 8).

When the passthrough client receives a call from another MCPTT client (i.e., the LTE system endpoint), it will auto-answer and pass all audio received to the local platform sound architecture. If the passthrough client detects an SU key from the LMR endpoint during an ongoing MCPTT call, the passthrough client will request floor control for the current call, arbitrated by the controlling MCPTT application server. At any time during the call, the passthrough client has the ability to request floor control if a radio key is detected.

\section{Test Bed}

The technologies discussed throughout Sec. 3, SDR, GR, and PJSIP, are brought together in the passthrough solution, implemented in the PSCR telecommunications laboratory as seen in Fig. 13. IP-based networking architecture provides interconnectivity to the different passthrough client technologies hosted in the test bed. IP security is ensured via a trusted networking segment, preventing any local or external data packets from exiting or being received by the system. All components of the PSCR test bed are discussed in detail in the following sections.

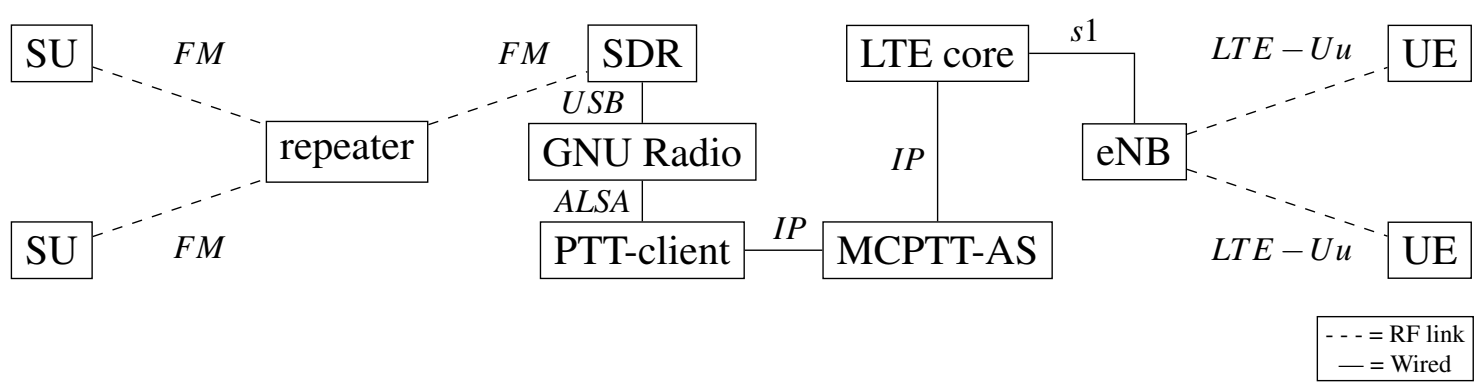

Fig. 13. Client-based proof of concept test bed

\subsection{LMR Hardware}

The test bed configuration includes a VHF/ UHF analog FM Kenwood TKR-740 repeaterbase unit. This unit provides connected SUs a single conventional analog FM LMR channel. In field implementations, an SU would communicate with the bridging SDR through 
an LMR repeater. The selection of LMR SUs used in conjunction with the repeater is relatively unimportant, provided they are compatible with the system. Some analog SUs can be programmed to use basic forms of in-band signaling to provide rudimentary data services, discussed in Sec. 5.1.3.

\subsection{MCPTT}

The MCPTT ecosystem is comprised of the MCPTT application server and MCx User Equipment (UE) clients. PSCR is outfitted with the Nemergent Solutions' MCPTT application server and MCx UE MCPTT client applications. Both of these Nemergent resources are 3GPP standards-compliant and updated as more 3GPP standard features are developed. All testing and verification has been carried out using the "Nemergent MCS Client" sample application and "Nemergent Provisioning Tool," whose interfaces are displayed in Fig. 14. The Nemergent MCPTT application server is implemented as both the participating and controlling servers in the PSCR lab.

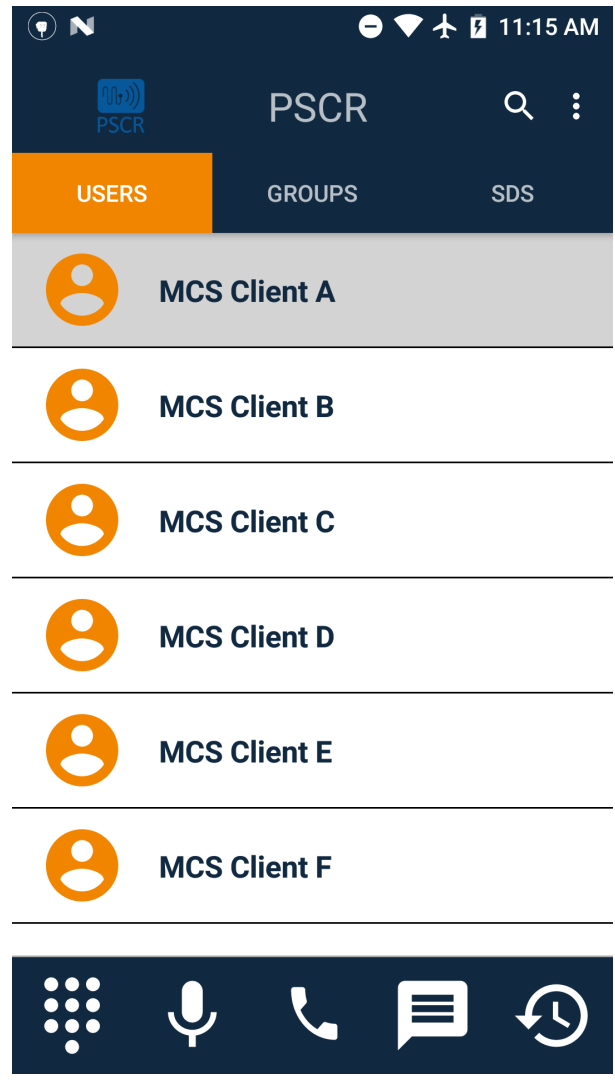

(a) MCS Client
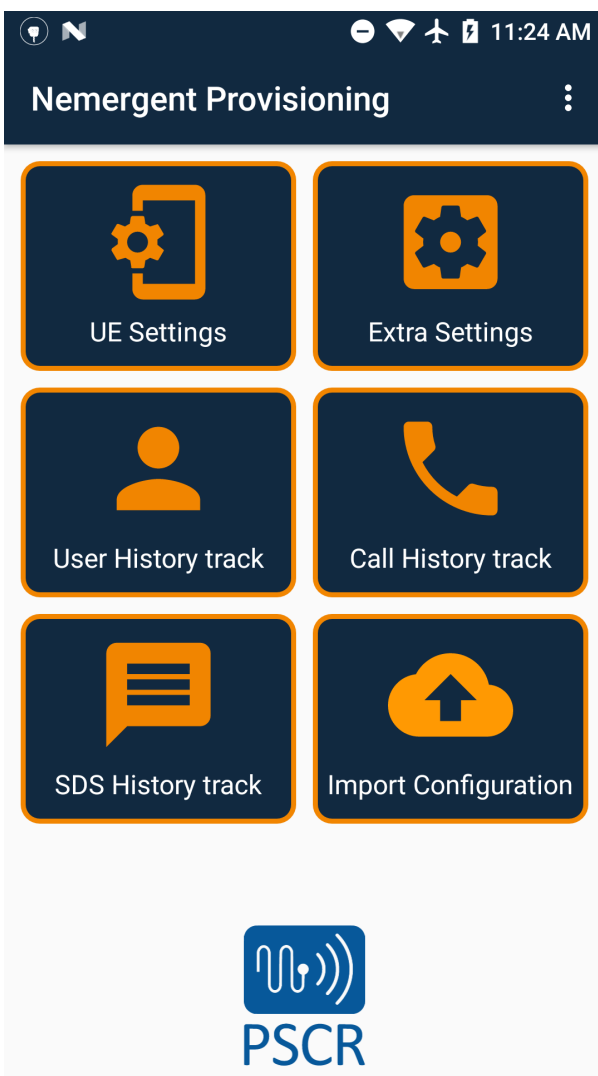

(b) Provisioning tool

Fig. 14. Nemergent UE MCPTT applications 


\subsubsection{Enabler}

In order to conduct analog FM LMR to MCPTT interworking research without a finalized L603 IWF interface standard, discussed in Sec. 2.3.4, it was necessary to develop an IWF workaround. Nemergent has developed a SIP-based workaround called the Enabler. The Enabler provides low-level IWF functionality through SIP messaging to the MCPTT application server. This allows nontraditional clients to register as non-MCx users and participate in private and group calls, request/release floor control, and transmit/receive MCData.

\subsection{LTE System Architecture Evolution}

The Evolved Packet Core (EPC) in PSCR's LTE test bed is implemented via a Polaris Networks NetEPC. A system diagram is shown in Fig. 15. The NetEPC allows for the logging of various EPC interfaces, enabling the debugging and development of MC client software. Visibility into Diameter, SIP and RTP messaging internal to the LTE system was essential during design phases. The PSCR test bed has additional logging capabilities which are available at the network level. The major focus during the development effort centered around SIP messaging between PJSIP stacks and the MCPTT application server. These tools were used to verify standards-based MC functionality, including messaging on the receiver interface and verification of requested and granted MC bearers during calls.

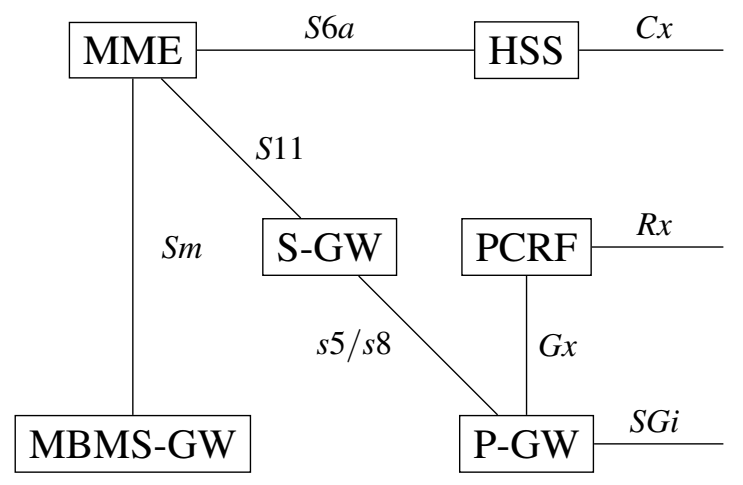

Fig. 15. Polaris EPC core architecture

The test bed has air interfaces to both the SU and UE endpoints. The LTE portion was developed with a Rel-14 3GPP compliant solution. The PSCR lab uses a set of fully 3GPP compliant Evolved UTRAN (E-UTRAN) base stations manufactured by major telecommunications vendors that supply many of the base stations commercially broadcasting in North America. These base stations provide a stable solution for testing and development. One possible shortcoming from utilizing commercial hardware is that the software releases available are limited to those that are used in commercial deployments. These field-deployed commercial software releases often do not implement newly defined 3GPP features. Software versions that support future feature sets exist in the vendors' development labs, but they are rarely released externally. 


\section{Results}

Research and development for the passthrough client was carried out with three metrics in mind: robustness, standards compliance, and low cost. A robust solution was achieved by using only stable published releases of established and credible open-source software. During development of this passthrough solution, care was taken only to implement standardscompliant software stacks whenever possible. The use of free and open-source software paired with SDR technology in place of normal donor radios provides a communications solution at a much lower price point. The passthrough client prototype defined herein successfully provides a bridging solution between analog FM LMR and MCPTT-enabled broadband LTE. Implementation of the prototype facilitates full-duplex PTT communications between these previously siloed communications systems. The passthrough client solution provides a simple feature set, supporting real-time group and private calls with floor control and priority.

Although the passthrough client provides a working proof of concept, it is far from a commercial product. The solution contains nontrivial user setup, does not support many MCx features outside of those discussed and has very limited OTA range, as no RF power amplifier has been implemented to augment the SDR. These shortcomings leave the door open to many different avenues of improvement. Overall, we view this outcome as a success, highlighting the merit of tools not yet found in the public safety ecosystem. Many of these issues could be remedied with the inclusion of 3GPP's IWF. As discussed in Sec. 5.1.4, implementing an OTA LMR passthrough client in conjunction with an IWF has the potential to simplify greatly current passthrough client complexity and introduce additional communications functionality called out in the MC 3GPP standards. Even taking into account drawbacks of the passthrough client solution, the proof of concept shows the possibility of new reliable standards-based low-cost communications interoperability solutions.

\subsection{Future Work}

While our efforts concerning audio passthrough from analog LMR to MCPTT LTE are successful at a basic level, there are many desirable additions that could be included in future revisions. High-cost and proprietary solutions are prohibitive for many public safety organizations, limiting their ability to operate and maintain strong and interoperable communications networks. One way to make solutions available for public safety agencies would be to create an open-source project providing public safety with an interoperability solution, as discussed in Sec. 5.1.1. One area of continued passthrough client development should be the improvement of the audio handling mechanism. The current usage of ALSA is undesirable, as further discussed in Sec. 5.1.2.

Analog LMR will never have the full feature set found in digital LMR systems. This feature gap could be partially remedied with the addition of analog system SUs that are capable of in-band signaling, further discussed in Sec. 5.1.3. The biggest challenge to this solution in the long term will be its integration with the upcoming 3GPP IWF. When 
implemented, the IWF will handle all encryption and transcoding as an endpoint or a passthrough. The IWF will also be the arbiter of floor control, priority, and user management. Implementation of the IWF in our test bed will greatly simplify the scope of the passthrough client and allow for a 3 GPP standard-compliant solution.

\subsubsection{Accessibility and Reliability}

In order for any interoperability solution to make a meaningful impact in the public safety community, it must be accessible and reliable. Given that the passthrough solution would not be the arbiter of any system security, utilizing an open-source solution should pose minimal operational security concerns. To maximize accessibility, the passthrough client software could be implemented using open-source code and placed in a public repository like GitHub or CGRAN. This would allow anyone with an internet connection access to a public code repository, facilitating a user community to maintain and update code. There are many examples of open-source projects that operate in this manner and have significant continuing impacts in the private and public sectors.

Prize challenges could be used to engage industry and academia to develop reliable open-source interoperability software. Open-source solutions with strong development communities are instrumental in keeping software up to date and reliable. There are many current examples of productive open-source software projects being run in this manner.

\subsubsection{Audio Handling}

A more commercially-ready solution should use GR network sinks in place of audio sinks to pass the GR channelized audio into the PJSIP-based client. A network transport method was not implemented in our proof of concept due to time constraints and additional software complexity. Using ALSA, the existing platform's audio infrastructure allows GR to handle multiple audio streams by creating additional virtual audio channels. This implementation allows the developer easy access to live audio communications while isolating additional channels from one another. Although functional, in future revisions audio should be handled using IP protocols, fully replacing ALSA. This change will greatly decrease user complexity and will simplify further research and development.

\subsubsection{In-Band Signaling with Analog FM}

Conventional analog FM LMR presents inherent limitations when compared to digital public safety communications systems. These limitations preclude many desirable public safety feature sets that are possible with digital systems, such as MCPTT. If in-band signaling is implemented on the analog LMR SUs, some functional parity can be regained. MCPTT communications features and their compatibility with different LMR systems is discussed at length in the NPSTC Interoperability report [2]. 


\subsubsection{IWF Integration}

The IWF is currently being defined to enable the interworking of LMR-based PTT systems with LTE-based MCPTT systems. As discussed in Sec. 2.3.4, 3GPP and TIA are working on a common IWF solution that will support the ISSI interface [24]. ISSI solutions were originally developed to be the TIA-defined wireline interface between RFSS. To support analog LMR, any ISSI-based interworking solution must have a way to provide audio and control signaling to and from an analog SU through an RFSS. Solutions for this purpose have been proposed by a Department of Homeland Security (DHS) Feasibility Study [25]. In contrast to the DHS solution, the PSCR passthrough client is seen by the MCPTT application server as an MCPTT non-MCx user. After successful registration, the passthrough client utilizes SIP and RTP directly with the MCPTT application server.

Once IWFs become standardized and deployed, the passthrough client developed herein will need to be enhanced to interface with an RFSS. Vocoding and floor control duties, currently handled by the passthrough client, will be migrated to the IWF. Integration of the passthrough client with an IWF could build on the InterWorking Group (IWG) adapter solution proposed in the Catalyst/DHS paper [25]. The flexibility of GR and SDR could allow modification of the PSCR passthrough client by allowing it to interface with an IWG adapter. This solution would decrease the complexity of the passthrough client by transferring feature functionality to the IWG adapter. The passthrough client currently relies on the Nemergent Enabler for floor control and non-MCx client registration, discussed in Sec. 4.2.1. A modification of the passthrough client that is needed to interface with an ISSI is the replacement of the VoIP-based SIP floor control messages. Updating the passthrough client to use standards-based RTCP floor control messages will allow operation with an IWF. 


\section{References}

[1] TIA (2016) Land Mobile FM or PM Communications Equipment Measurement and Performance Standards. Telecommunications Industry Association (TIA), Standard 102.E.

[2] National Public Safety Telecommunications Council (2018) Public Safety Land Mobile Radio (LMR) Interoperability with LTE Mission Critical Push to Talk. National Public Safety Telecommunications Council (NPSTC), Technical Report Final Report.

[3] 3GPP (2015) Overview of 3GPP Release 13. 3rd Generation Partnership Project (3GPP), Overview 0.0.1. URL https://www.3gpp.org/ftp/Information/WORK_PLAN/ Description_Releases/Rel-13_description_20150917.zip.

[4] 3GPP (2014) Overview of 3GPP Release 14. 3rd Generation Partnership Project (3GPP), Overview 0.0.1. URL https://www.3gpp.org/ftp/Information/WORK_PLAN/ Description_Releases/Rel-14_description_20140924.zip.

[5] 3GPP (2019) Mission Critical Push to Talk (MCPTT). 3rd Generation Partnership Project (3GPP), Technical Specification (TS) 21.915. Version 15.0.0 URL https:// www.3gpp.org/ftp/Information/WORK_PLAN/Description_Releases/21915-f00.zip.

[6] USA (2012) Middle Class Tax Relief And Job Creation Act Of 2012. URL www. congress.gov/112/plaws/pub196/PLAW-112pub196.pdf.

[7] Mission Critical Communications (2020) Public Safety Advocate: Another MCPTT Vendor? FirstNet Coverage, Drive Tests. URL https://allthingsfirstnet.com/ public-safety-advocate-another-mcptt-vendor-firstnet-coverage-drive-tests/.

[8] Mission Critical Communications (2018) 4 Suppliers Contribute to AT\&T's Contract with FirstNet. URL https://www.rrmediagroup.com/Features/FeaturesDetails/ FID/927.

[9] Firstnet (2019) FirstNet Authority ROADMAP. FirstNet, Roadmap. URL https: //www.firstnet.gov/sites/default/files/FirstNet_Roadmap.pdf.

[10] Networks PSW (2002) Software Enabled Wireless Interoperability Assessment Report—Software Define Radio Subscriber Equipment. URL https://www.hsdl.org/ ?view\&did=14719.

[11] TIA (2017) Tia standard;project 25; fixed station interface messages and procedures.

[12] PSTA (2019) LMR LTE Interoperability Technical Subcommittee Report https://www.pstalliance.org/wp-content/uploads/PSTA-LMRLTE-InteroperabilityTechnical-Subcommittee-Report.pdf.

[13] 3GPP (2019) Mission critical communication interworking with land mobile radio systems. URL https://www.3gpp.org/ftp/Specs/archive/23_series/23.283/.

[14] 3GPP (2017) Study on mission critical communication interworking between lte and non-lte systems. URL https://portal.3gpp.org/desktopmodules/Specifications/ SpecificationDetails.aspx?specificationId $=3083$.

[15] 3GPP (2018) Lte;mission critical communication interworking with land mobile radio systems (3gpp ts 23.283 version 15.1.0 release 15). URL https://www.etsi.org/deliver/ etsi_ts/123200_123299/123283/15.01.00_60/ts_123283v150100p.pdf. 
[16] FCC (2016) Narrowbanding Overview https://www.fcc.gov/narrowbandingoverview.

[17] Public Safety Communications Online (2011) 730 Days and Counting. URL https: //psc.apcointl.org/2011/01/20/730-days-counting/.

[18] FCC (2018) Public Safety Spectrum https://www.fcc.gov/public-safety/public-safetyand-homeland-security/policy-and-licensing-division/public-safety-spectrum.

[19] Ettus (2020) UB210-kit https://www.ettus.com/all-products/ub210-kit/.

[20] The comprehensive gnu radio archive network (cgran). URL https://cgran.org.

[21] Blossom E (2020) Outoftreemodules. URL https://wiki.gnuradio.org/index.php/ OutOfTreeModules.

[22] Smith C (2020) Gr-dsd. URL https://www.cgran.org/1291/.

[23] PJSIP (2020) SM Windows NT kernel. URL https://pjsip.org.

[24] 3GPP (2019) Mission Critical Communications Interworking with Land Mobile Radio Systems. 3rd Generation Partnership Project Sophia Antipolis, FR, Technical Specification (TS) 3GPP TS 23.283 v16.3.0 2019-06.

[25] Catalyst Communications Technologies Inc (2019) Interworking MCPTT between LTE and LMR. 